\title{
Rheumatoid Arthritis in the Lebanese Adults: Impact on Health-Related Quality of Life
}

\author{
Sanaa Awada*, Roula Ajrouche, Mariam Shoker, Amal Al-Hajje, Samar Rachidi, Salam Zein, Wafaa Bawab \\ Clinical and Epidemiological Research Laboratory, Faculty of Pharmacy, Lebanese University, Hadath, Lebanon
}

\section{ARTICLE INFO}

Article History

Received 03 February 2019

Accepted 19 August 2019

\section{Keywords}

Rheumatoid arthritis quality of life

health-related quality of life

SF-36

Lebanese population

\begin{abstract}
Rheumatoid Arthritis (RA) is a chronic inflammatory disabling disease with significant impact on the Quality of Life (QOL) of patients. Information on the effects of RA on Health-related Quality of Life (HRQoL) is lacking in the Lebanese population. The objective of this study was to evaluate QOL of RA patients compared with non-RA subjects and to suggest possible predictors of their QOL in Lebanon. We conducted a case-control observational study among individuals visiting the external clinics at three hospitals and different private clinics; the QOL was measured using the SF-36 questionnaire administered face to face to the study population, applied to RA $(N=90)$ and non-RA $(N=180)$ groups. RA presented lower Physical Component Scores (PCS) and Mental Component Scores (MCS) as well as overall QOL scores. Among RA patients, MCS and QOL were significantly decreased with morning stiffness duration $(\beta=-9.211, p=0.013$ and $\beta=-9.190, p=0.009$, respectively). The frequency of practicing sport per week increases PCS and QOL $(\beta=6.692, p=0.002$ and $\beta=6.148, p=0.003$, respectively). Workability has a positive effect on PCS $(\beta=5.546, p=0.022)$ and time between blood transfusion and the onset of the disease has a positive impact on MCS $(\beta=8.415, p=0.007)$. To improve QOL of patients with RA, health professionals have to take these results into consideration while treating their patients.
\end{abstract}

(c) 2019 Atlantis Press International B.V.

This is an open access article distributed under the CC BY-NC 4.0 license (http://creativecommons.org/licenses/by-nc/4.0/).

\section{INTRODUCTION}

Rheumatoid arthritis (RA) is a chronic inflammatory autoimmune disease characterized by pain, stiffness, swelling, and tenderness of synovial joints [1]. There is an estimated prevalence of RA in the world population from 0.5 to $1 \%$, with mean annual incidence of $0.02-0.05 \%$. Women are more likely to develop RA than men [2]. Predominance is two to three times higher in females, whose peak is observed around the age of 50 years [3]. Being overweight increases the risk of RA for women but actually decreases the risk for men [2]. Prolonged smoking has a role in increasing the concentration of rheumatoid factor, "which is an antibody". Moderate consumption of alcohol does not seem to increase women's risk and may even lower it. In addition, specific diet rich in antioxidants and poor in red meat and sugars may decrease the risk of RA [4].

According to the World Health Organization (WHO), quality of life (QOL) is defined as "the individuals' perceptions of their position in life in the context of the culture and value systems in which they live and in relation to their goals, expectations, standards and concerns" [5]. Ever since this definition was given by the WHO in 1948, there has been a major emphasis on the impact of diseases on the QOL of patients [5]. Health-related quality of life (HRQoL) refers to the extent to which patient's usual or expected physical, emotional, and social well-being are influenced by their medical condition and/or by the treatment they receive $[3,6,7]$. QOL is a concept increasingly

Corresponding author.Email: sanaa3a@hotmail.com; s.awada@ul.edu.lb used in the assessment of health care outcome of a given population (diet habits, housing, employment, recreation, and so on) and of the impact of therapeutic applications associated with different groups of diseases [3]. Historically, the impact of chronic diseases on the patients' lives has been defined in terms of three different levels stated by the WHO: impairment, disability, and handicap [8]. As other chronic diseases, RA is a chronic inflammatory disabling disease with significant impact on the QOL of patients [5,9]. Although RA primarily affects the joints and leads to physical impairment, many patients with RA also experience psychological effects such as: depression, anxiety, feelings of helplessness, low self-esteem, loss of energy, and fatigue $[1,2,8,10]$. Pain and stiffness can last for more than $30 \mathrm{~min}$ in the morning or after a long period of rest. Higher pain levels in patients with RA have been shown to correlate with disability as well as depression, which all contribute significantly to a reduction in QOL $[2,8]$. Besides, permanent work disability and instability are common among patients with RA and have a significant impact on patients' financial and social well-being that may lead to QOL deterioration $[6,11,12]$.

For the Lebanese population, the prevalence of rheumatic diseases was found to be about $15 \%$ by Chaaya et al. [6]. Nevertheless, no study has been previously conducted regarding the HRQoL of RA patients and its significance in the Lebanese population. As "adding years to life is an empty victory without adding life to years" as WHO states [13], the objective of this study was to evaluate QOL of RA patients compared with non-RA patients in Lebanon and to suggest possible predictors of QOL in the two groups. 


\section{MATERIALS AND METHODS}

\subsection{Study Design}

This is an observational case-control study carried out in RA patients undergoing treatment and non-RA patients. A convenient sample of patients was randomly selected from those visiting external clinics at three hospitals (AL Rasool Hospital, Bahman Hospital, and Saint George Hospital), and patients visiting rheumatologists' private clinics in Beirut, Lebanon. Cases and controls were recruited based on the presence or absence of RA as a diagnosis in their medical files.

The scheme of this study maintains the total anonymity of the patients and does not violate human rights. Even though it was not an interventional study and therefore did not require any definite ethical approval, however, verbal consent was obtained from all participants before administering the questionnaire, which was completed in the waiting room before the clinical assessment. An approval was taken from the Institutional Review Board to carry out the study at each hospital as well as from each doctor to carry out the study at his/her clinic. The patients were informed about the objective of the study and asked to provide an oral consent before they started with the procedure. Data were collected from August until the end of September 2017.

\subsection{Study Population}

All outpatients aged 32-60 years of both genders with confirmed RA disease duration of 1-20 years were recruited as cases. The diagnosis had to be confirmed by a clinical rheumatologist. Patients who did not have any official confirmation of RA, heavily handicapped patients, patients with language limitations (who did not speak Arabic or English), non-Lebanese patients, pregnant women, patients who did not provide verbal consent, as well as patients suffering from neurological problems were all excluded from the study.

Controls were selected by simple random sampling from the patients aged 32-60 years of both genders who were visiting outpatient clinics situated close to rheumatology clinic at the same study period: patients attending clinics of respiratory, endocrinology, urology, cancer, pre-surgery consultation, and gastroenterology. For every case included in the study up to two controls were recruited.

\subsection{Data Collection}

Data were acquired through a structured face-to-face interview. The questionnaire was administered in Arabic, the main language in Lebanon, and included: sociodemographic characteristics of patients including sex, age, education level, marital status, monthly income, Body Mass Index (BMI), district of residence, employment and work demand (mental, physical, or both physical), work disability, and the source of insurance; life style data such as smoking, alcohol consumption, and physical exercise. Detailed health history was also included in relation with the onset of RA, the presence of associated complications and comorbidity (e.g., cardiovascular diseases, diabetes mellitus, psychological disorders, obstructive pulmonary disease, osteoporosis, and so on), and medication use.

\subsection{QOL Measurement}

The QOL of patients was assessed using an Arabic version of the SF-36 generic questionnaire as described by Sabbah et al. [7]. It consists of 36 questions that are distributed across eight scales: Physical Functioning (PF) (10 items), Role Physical (RP) limitation (four items), Bodily Pain (BP) (two items), General Health (GH) (five items), Vitality (VT) (four items), Social Functioning (SF) (two items), Role Emotional (RE) limitation (three items), and Mental Health (MH) (five items). From these eight scales, it is possible to calculate two summary scores, Physical Component Summary (PCS - physical functioning, role physical limitation, bodily pain, and general health), which takes into account all aspects of the physical daily activity or at work outside home, and Mental Component Summary (MCS - social functioning, role emotional limitation, RA mental health, and vitality), and the overall QOL. The answer for each question corresponds to a score as a percentage as stated by Hays et al. [9]. Data are presented as means; statistical significance was tested using $t$-test, all $p$-values $<0.05$ are statistically significant. Higher scores represent higher levels of functioning or well-being.

\subsection{Sample Size}

Sample size was calculated using Epi Info 7, assuming a type I error of $5 \%$, study power of $80 \%$, and confidence interval of $95 \%$. Taking Lebanese RA prevalence as $14.3 \%$ [12], the result was 90 cases and 180 controls with cases/control ratio of $1 / 2$. Hence, the total study population size was 270 participants. This minimal sample size was necessary to show a threefold increase in the risk for most of the associations with frequency of exposure about 0.6 in the control group.

\subsection{Statistical Analysis}

Data were entered and analyzed using Statistical Package for Social Sciences (SPSS, IBM, USA), version 21. No missing data were obtained. A $p$-value $\leq 0.05$ was considered significant in all tests. Descriptive analysis was done first to evaluate the distribution of the sociodemographic characteristics of the patients, their life style behaviors, and their past medical history. The results are expressed in frequency and percentage $[n(\%)]$ for all the qualitative variables and in mean $( \pm)$ Standard Deviation (SD) for quantitative variables. An appropriate bivariate analysis was done for every explanatory variable. For comparing continuous variables, we used Student's $t$-test or one-way ANOVA for variables with adequate normal distribution to compare between mean. For non-normally distributed continuous variables, the Mann-Whitney U or Kruskal-Wallis test was used. For categorical variables, the Chisquare and Fisher exact tests were used to compare percentages.

Similarly, a multivariable analysis using linear regression was carried out to determine predictive factors affecting the QOL. The major dependent variables were PCS scores, MCS scores, and the overall QOL, which is the sum of all individual domains of SF-36 items (physical functioning, physical role limitation, pain, general health perception, vitality, social functioning, emotional role limitation, and mental health) in addition to one item to compare current health status and that of the previous year (perceived health change). The analysis sought to explore factors associated with general physical and mental health using regression analysis. However, the independent variables are all potential confounding variables that had 
$p$-value $<0.2$ in bivariate analysis including: sociodemographic, life style, adherence, patient health, and disease status. Conditions of normality, linearity, adequacy, and homoscedasticity for data were checked for the linear regressions using Hosmer and Lemeshow test.

\section{RESULTS}

Only 270 patients were solicited to fill the questionnaire. All patients agreed to participate in this study. Among them, 90 patients $(33.3 \%)$ diagnosed with RA were included as cases and 180 patients $(66.7 \%)$ who were not diagnosed with RA were included in the control group.

\subsection{Sociodemographic Characteristics of the Study Population}

The comparison of characteristic differences between RA and control groups is shown in Table 1 . Female patients (62.2\%) were significantly more affected by RA than male patients $(37.8 \%, p=0.025)$. Patients with RA were older than control group $(p=0.02)$. Significant difference was found between RA and control in the place of living $(p<0.001)$, such that most of the cases were found living in Beirut (77.8\% versus $62.8 \%$ of the controls), $7.8 \%$ of the cases were living in Bekaa and $7.8 \%$ in South Lebanon, whereas it was $22.8 \%$ and $14.4 \%$ for the control group, respectively. Greater number of jobless participants were found in RA group $(p=0.031)$. There was no significant difference between RA and controls regarding marital status $(p=0.059)$, education level, monthly income, and the type of medical insurance $(p>0.05)$.

\subsection{Behavioral Characteristics}

Concerning lifestyle factors and habits (Table 2), a significant difference was found between RA and controls regarding smoking $(p=0.048)$ and alcohol consumption $(p=0.033)$, knowing that the majority of the participants did not consume alcohol, were significantly higher for controls than for cases. In addition, practicing sport regularly $(p=0.04)$, frequency of practicing sport $(p<0.001)$, and duration of sport each time $(p=0.015)$ were significantly higher for cases than for controls. Nonsignificant difference was found between cases and controls regarding BMI, specific diet, and smoking $(p>0.05)$.

\subsection{Medical and Medication History}

Table 3 shows that significant differences were found between RA patients and controls in case of: medication taken, suffering from at least one comorbid condition, duration of morning stiffness $(p<0.001)$, and taking drug regularly $(p=0.045)$. Nonsignificant difference was found between the two groups regarding the following variables: perform lab test regularly and regular physician visit $(p>0.05)$.

\subsection{Health-related Quality of Life}

Figure 1 shows differences in SF-36 dimension scores and total QOL between cases and controls. This bivariate analysis showed
Table $1 \mid$ Rheumatoid arthritis and related sociodemographic variables

\begin{tabular}{|c|c|c|c|}
\hline Variables & $\mathbf{R A}^{*}[n(\%)]$ & Control $[n(\%)]$ & $p$-Value \\
\hline Sex & & & 0.025 \\
\hline Male & $34(37.8)$ & $94(52.2)$ & \\
\hline Female & $56(62.2)$ & $86(47.8)$ & \\
\hline Age & & & 0.020 \\
\hline $24-35$ & $23(25.6)$ & $48(26.7)$ & \\
\hline $36-50$ & $39(43.3)$ & $47(26.1)$ & \\
\hline$>50$ & $28(31.1)$ & $85(47.2)$ & \\
\hline District & & & $<0.001$ \\
\hline Beirut & $70(77.8)$ & $113(62.8)$ & \\
\hline Bekaa & $7(7.8)$ & $41(22.8)$ & \\
\hline South & $7(7.8)$ & $26(14.4)$ & \\
\hline North & $6(6.7)$ & $0(0)$ & \\
\hline Marital status & & & 0.059 \\
\hline Single & $17(18.9)$ & $18(10)$ & \\
\hline Married & $64(71.0)$ & $150(83.3)$ & \\
\hline Divorced & $9(10)$ & $12(6.7)$ & \\
\hline Education level & & & 0.258 \\
\hline Not educated & $16(17.8)$ & $49(27.2)$ & \\
\hline Primary school & $32(35.6)$ & $49(27.2)$ & \\
\hline Middle education & $19(21.1)$ & $42(23.3)$ & \\
\hline High education & $23(25.6)$ & $40(22.2)$ & \\
\hline Occupation & & & 0.031 \\
\hline Jobless & $49(54.4)$ & $74(41.1)$ & \\
\hline Employee & $33(36.7)$ & $70(38.9)$ & \\
\hline Retired & $8(8.9)$ & $36(20)$ & \\
\hline Work demand & & & 0.260 \\
\hline No & $46(51.1)$ & $108(60)$ & \\
\hline Mental & $10(11.1)$ & $21(11.7)$ & \\
\hline Physical & $17(18.9)$ & $32(17.8)$ & \\
\hline Mental + physical & $17(18.9)$ & $19(10.6)$ & \\
\hline Work ability & & & 0.152 \\
\hline No & $32(35.6)$ & $59(32.8)$ & \\
\hline Fully & $20(22.2)$ & $60(33.3)$ & \\
\hline Partially & $38(42.2)$ & $61(33.9)$ & \\
\hline Income (LP/Month) & & & 0.080 \\
\hline 0 & $48(53.3)$ & $76(42.2)$ & \\
\hline$<10,00,000$ & $21(23.3)$ & $38(21.1)$ & \\
\hline$\geq 10,00,000$ & $21(23.3)$ & $66(36.7)$ & \\
\hline Insurance & & & 0.157 \\
\hline No & $29(32.2)$ & $71(39.4)$ & \\
\hline Social security & $21(23.3)$ & $27(15)$ & \\
\hline Cooperative staff & $6(6.7)$ & $17(9.4)$ & \\
\hline Insurance & $6(6.7)$ & $7(3.9)$ & \\
\hline Ministry of Public Health & $22(24.4)$ & $34(18.9)$ & \\
\hline Military & $6(6.7)$ & $24(13.3)$ & \\
\hline Total $(N)$ & $90(33.3)$ & $180(66.7)$ & \\
\hline
\end{tabular}

"RA, rheumatoid arthritis; [ $n(\%)]$, frequencies (percentages). $p<0.05$ is considered significant, 10,00,000 LP (Lebanese pound) $\simeq 650$ USD.

that for four dimensions (PF, RP, RE, and VT), the two scores PCS $(p<0.001)$ and MCS $(p=0.031)$, and the total QOL $(p<0.001)$ were significantly lower in RA patients than in controls. However, $\mathrm{GH}$ was significantly higher for cases than for controls $(p=0.04)$. Nonsignificant difference was found regarding $\mathrm{MH}(P=0.45), \mathrm{SF}$ $(p=0.967)$, and BP $(p=0.156)$.

\subsection{Intragroup Analysis of QOL and its Components among Control Group}

In the multivariable linear regression model estimating the predictor of PCS (Table 4), we found that age $[\beta=-6.750, p<0.001,95 \%$ 
Table 2 Rheumatoid arthritis and lifestyle-related variables

\begin{tabular}{|c|c|c|c|}
\hline Variables & $\mathbf{R A}^{*}[n(\%)]$ & Control $[n(\%)]$ & $p$-Value \\
\hline BMI & & & 0.262 \\
\hline Underweight & $12(13.3)$ & $33(18.3)$ & \\
\hline Normal & $27(30)$ & $37(20.6)$ & \\
\hline Overweight & $30(33.3)$ & $72(40)$ & \\
\hline Obese & $21(23.3)$ & $38(21.1)$ & \\
\hline Specific diet & & & 0.900 \\
\hline No & $36(40)$ & $72(40)$ & \\
\hline Yes & $54(60)$ & $108(60)$ & \\
\hline Smoking & & & 0.161 \\
\hline No & $54(60)$ & $98(54.4)$ & \\
\hline Yes & $27(30)$ & $72(40)$ & \\
\hline Past smoker & $9(10)$ & $10(5.6)$ & \\
\hline $\begin{array}{l}\text { Number of } \\
\text { cigarettes/day }\end{array}$ & & & 0.048 \\
\hline 0 & $53(58.9)$ & $98(54.4)$ & \\
\hline$<10$ & $6(6.7)$ & $23(12.8)$ & \\
\hline $10-20$ & $11(12.2)$ & $38(21.2)$ & \\
\hline $20-40$ & $9(10)$ & $11(6.1)$ & \\
\hline$>40$ & $11(2.2)$ & $10(5.6)$ & \\
\hline Alcohol & & & 0.033 \\
\hline No & $86(95.6)$ & $167(92.8)$ & \\
\hline Yes & $4(4.4)$ & $13(5.6)$ & \\
\hline Sport & & & 0.040 \\
\hline No & $56(62.2)$ & $133(73.9)$ & \\
\hline Yes & $34(37.8)$ & $47(26.1)$ & \\
\hline Sport (times/week) & & & $<0.001$ \\
\hline 0 & $47(52.2)$ & $121(67.2)$ & \\
\hline$<3$ & $14(15.6)$ & $38(21.1)$ & \\
\hline$>3$ & $29(32.2)$ & $21(11.7)$ & \\
\hline $\begin{array}{l}\text { Duration of sport } \\
\text { each time (min) }\end{array}$ & & & 0.015 \\
\hline 0 & $47(52.2)$ & $121(67.2)$ & \\
\hline$\leq 30$ & $21(23.3)$ & $38(21.1)$ & \\
\hline$>30$ & $22(24.4)$ & $21(11.7)$ & \\
\hline Total & $90(33.3)$ & $180(66.7)$ & \\
\hline
\end{tabular}

*RA, rheumatoid arthritis; $[n(\%)]$, frequencies (percentages); BMI, body mass index. $p<0.05$ is considered significant.

confidence interval (CI): $(-10.292 ;-3.207)]$, BMI $[\beta=-2.907$, $p=0.036,95 \% \mathrm{CI}:(-5.617 ;-0.197)]$, and morning stiffness $\geq 10 \mathrm{~min}$ $[\beta=-26.366, p=<0.001,95 \% \mathrm{CI}:(-37.575 ;-15.158)]$ were negatively associated with PCS. Number of cigarettes per day $[\beta=3.575$, $p=0.001,95 \%$ CI: $(1.453 ; 5.696)]$, education level $[\beta=3.425, p=$ $0.030,95 \% \mathrm{CI}:(0.345 ; 6.506)]$, and workability $[\beta=5.143, p=0.003$, 95\% CI: $(1.738 ; 8.548)]$ were positively associated with PCS.

In the multivariable linear regression model estimating the predictor of MCS (Table 4), our results show that morning stiffness $[\beta=-25.009, p<0.001,95 \% \mathrm{CI}:(-38.718 ;-11.200)]$ and comorbidity $[\beta=-35.941, p=0.004,95 \% \mathrm{CI}:(-59.988 ;-11.895)]$ are negatively associated with MCS. However, income $[\beta=4.727$, $p=0.037,95 \% \mathrm{CI}:(0.278 ; 9.175)]$ and work ability $[\beta=4.991$, $p=0.019$, 95\% CI: $(0.826 ; 9.155)]$ positively affect MCS.

In addition, we found that age $[\beta=-30.790, p=0.006,95 \% \mathrm{CI}$ : $(-52.710 ;-8.860)]$, morning stiffness $[\beta=-25.688, p<0.001,95 \%$ CI: $(-34.568 ;-16.807)]$, and comorbidity $[\beta=-27.552, p=0.001$, 95\% CI: $(-43.128 ;-11.975)]$ are negatively associated with overall QOL score. However, number of cigarettes per day $[\beta=1.931, p=$ $0.025,95 \% \mathrm{CI}:(0.251 ; 3.612)]$ and workability $[\beta=5.067, p<0.001$, $95 \%$, CI: $(2.370 ; 7.765)]$ are positively associated with QOL (Table 4$)$.
Table 3 Rheumatoid arthritis and health-related variables

\begin{tabular}{lccc}
\hline Variables & RA $^{*}[\boldsymbol{n}(\%)]$ & Control $[\boldsymbol{n}(\%)]$ & $\boldsymbol{p}$-Value \\
\hline Medication taken & & & $<0.001$ \\
Rheumatoid drugs & $88(97.8)$ & $0(0)$ & \\
Other drugs & $2(2.2)$ & $180(100)$ & 0.045 \\
Taking drug regularly & & & \\
No & $7(7.8)$ & $30(16.7)$ & \\
Yes & $83(92.2)$ & $150(83.3)$ & \\
Perform lab test & & & \\
$\quad$ regularly & & & \\
No & $21(23.3)$ & $55(30.6)$ & \\
Yes & $69(76.7)$ & $125(69.4)$ & \\
Regular physician & & & \\
$\quad$ consultation & & & \\
No & $20(22.2)$ & $70(77.8)$ & \\
Yes & $42(23.3)$ & $138(76.7)$ & \\
At least one & & & \\
$\quad$ comorbidity & & & \\
No & $55(61.1)$ & $5(2.8)$ & \\
Yes & $35(38.9)$ & $175(97.2)$ & \\
Duration of morning & & & \\
stiffness (min) & & & \\
0 & $1(1.1)$ & $137(96.1)$ & \\
$<10$ & $44(48.9)$ & $4(2.2)$ & \\
$10-30$ & $45(50)$ & $3(1.7)$ & \\
Total & $90(33.3)$ & $180(66.7)$ & \\
\hline & & & \\
\hline
\end{tabular}

'RA, rheumatoid arthritis; [ $n(\%)]$, frequencies (percentages). a Cardiovascular diseases, diabetes mellitus, psychological disorders, cancer, obstructive pulmonary disease, and osteoporosis. $p<0.05$ is considered significant.

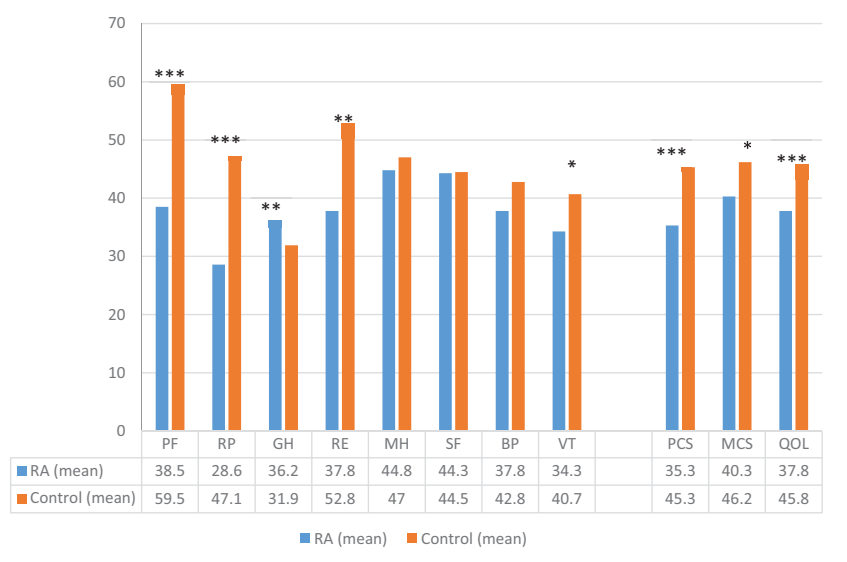

Figure $1 \mid$ Mean scores of the eight dimensions, physical and mental component summaries, and the overall score of quality of life of the SF36, comparing RA and control groups. ${ }^{*} 0.01 \leq p<0.05,{ }^{* *} 0.001 \leq p<0.01$, ${ }^{* * *} p<0.001$ are statistically significant. BP, body pain; GH, general health perception; MCS, mental component; $\mathrm{MH}$, mental health; QOL, quality of life; PCS, physical component; PF, physical functioning; RA, rheumatoid arthritis; RE, emotional role limitation; RP, physical role limitation; SF, social functioning; VT, vitality. Data are presented as means; statistical significance was tested using $t$-test.

\subsection{Intragroup Analysis of QOL and its Components among RA Group}

In the multivariable linear regression model estimating the predictor of PCS (Table 5), we found that the number of times of sport 
Table 4 Multivariable analysis of QOL and its components among controls $(N=180)$

\begin{tabular}{lcccr}
\hline Predictors & Standardized $\boldsymbol{\beta}$ & Unstandardized $\boldsymbol{\beta}$ & $\mathbf{9 5 \%} \mathbf{C I}^{*}$ & $\boldsymbol{p}$-Value \\
\hline PCS & & & & \\
Age & -0.282 & -6.750 & $-10.292 ;-3.207$ & $<0.001$ \\
Number of cigarettes per day & 0.219 & 3.575 & $1.453 ; 5.696$ & 0.001 \\
BMI & -0.147 & -2.907 & $-5.617 ;-0.197$ & 0.036 \\
Education level & 0.190 & 3.425 & $0.345 ; 6.506$ & 0.030 \\
Workability & 0.210 & 5.143 & $1.738 ; 8.548$ & 0.003 \\
Morning stiffness & -0.387 & -26.366 & $-37.575 ;-15.158$ & $<0.001$ \\
MCS & & & & \\
Income & 0.202 & 4.727 & $0.278 ; 9.175$ & 0.037 \\
Workability & 0.197 & 4.991 & $0.826 ; 9.155$ & 0.019 \\
Morning stiffness & -0.354 & -25.009 & $-38.718 ;-11.200$ & $<0.001$ \\
Comorbidity & -0.285 & -35.941 & $-59.988 ;-11.895$ & 0.004 \\
Overall QOL & & & & \\
Age & -0.198 & -3.533 & $-6.340 ;-0.726$ & 0.014 \\
Number of cigarettes per day & 0.159 & 1.931 & $0.251 ; 3.612$ & 0.025 \\
Workability & 0.278 & 5.067 & $2.370 ; 7.765$ & $<0.001$ \\
Morning stiffness & -0.505 & -25.688 & $-34.568 ;-16.807$ & $<0.001$ \\
Comorbidity & -0.304 & -27.552 & $-43.128 ;-11.975$ & 0.001 \\
\hline
\end{tabular}

"CI, confidence interval; $\beta$, regression coefficient; BMI, body mass index; MCS, mental component score; PCS, physical component score; QOL, quality of life. $p<0.05$ is considered significant.

Table 5 Multivariable analysis of QOL and its components among cases $(N=90)$

\begin{tabular}{|c|c|c|c|c|}
\hline Predictors & Standardized $\beta$ & Unstandardized $\beta$ & $95 \% \mathrm{CI}^{*}$ & $p$-Value \\
\hline \multicolumn{5}{|l|}{ PCS } \\
\hline Sport times per week & 0.324 & 6.692 & $2.619 ; 10.766$ & 0.002 \\
\hline Workability & 0.264 & 5.546 & $0.812 ; 10.281$ & 0.022 \\
\hline \multicolumn{5}{|l|}{ MCS } \\
\hline $\begin{array}{l}\text { Time between blood transfusion } \\
\text { and onset of the disease }\end{array}$ & 0.257 & 8.415 & $2.340 ; 14.490$ & 0.007 \\
\hline Morning stiffness & -0.227 & -9.211 & $-17.773 ;-0.650$ & 0.013 \\
\hline \multicolumn{5}{|l|}{ Overall QOL } \\
\hline Sport times/week & 0.312 & 6.148 & $2.214 ; 10.081$ & 0.003 \\
\hline Morning stiffness & -0.272 & -9.190 & $-16.027 ;-2.353$ & 0.009 \\
\hline
\end{tabular}

"CI, confidence interval; $\beta$, regression coefficient; MCS, mental component score; PCS, physical component score; QOL, quality of life. $p<0.05$ is considered significant.

activity per week $[\beta=6.692, p=0.002,95 \% \mathrm{CI}:(2.619 ; 10.766)]$ and work ability $[\beta=5.546, p=0.022,95 \% \mathrm{CI}:(0.812 ; 10.281)]$ are positively associated with PCS. The lapse of time between blood transfusion and onset of the disease $[\beta=-9.211, p=0.013,95 \%$ CI: $(-17.773 ;-0.650)]$ is positively associated with MCS; however, morning stiffness $[\beta=8.415, p=0.007,95 \% \mathrm{CI}:(2.340 ; 14.490)]$ is negatively associated with MCS (Table 5).

Table 5 also shows that the number of times of sport activity per week $[\beta=6.148, p=0.003,95 \% \mathrm{CI}:(2.214 ; 10.081)]$ is positively associated with the overall QOL among RA patients; however, morning stiffness is negatively associated with the overall QOL $[\beta=-9.190, p=0.009,95 \% \mathrm{CI}:(-16.027 ;-2.353)]$.

\section{DISCUSSION}

Quality of life has been the objective of several studies evaluating the impact of chronic diseases [14], cardiovascular diseases [12,13], dyslipidemia [15], and hypertension [16,17]. Any disease, especially chronic, affects QOL, physical, emotional, and social wellbeing, and the QOL measure is considered an important factor in the assessment of an individual's health status. RA is a preventable, autoimmune, systemic, and chronic disease. Many studies have evaluated the impact of RA on QOL worldwide [18]; however, there is lack of information in this field in Lebanon.

This is the first study conducted in Lebanon using case-control design to evaluate the impact of RA on the Lebanese patients' QOL and to suggest possible predictors of their QOL. Controls were selected using some similar criteria as cases and identified during the same time period as the cases. As age and gender are two variables highly associated with changes in QOL, our controls were not matched on these two variables to prevent overmatching that can lead to biased results. In addition, cases were recruited based on diagnosis confirmed by a clinical rheumatologist. However, our study was limited by the fact that the severity of disease and the details on treatments received by the patients, as well as their impacts on QOL, were not taken into account.

In our study, we have used the SF-36 health survey, which is a reliable tool for the assessment of the QOL in Lebanon $[15,16]$ and to assess the HRQoL of patients with RA [18]. Our results indicate that females were more affected by RA than males. These results are similar to those from other studies that demonstrated that women are more likely to develop RA than men and it is two to 
three times more prevalent in women than in men $[2,10]$. Most of RA patients in our study were aged between 36 and 50 years. A study conducted in a Dutch nationwide group of 1056 patients showed that the onset of RA reaches a peak between the ages of 40 and 50 years [10].

Suffering from RA deteriorates the physical health status as was seen in a descriptive study with 53 patients from a public rheumatology center in Montevideo, Uruguay. The study showed that RA is associated with higher disease burden, reflected on pain, impact on global health, and functional and working status, as well as the physical and emotional dimensions of the HRQoL [19].

In this study, RA had a negative influence on both physical $(p<0.001)$ and mental components $(p=0.031)$, where PCS and MCS for cases were lower than those in controls. These findings are consistent with a US study, where RA patients were taken as civilian non-institutionalized adults aged 18 years or older from 15 states, and conducted over a large population, participants with RA reported lower scores on the overall health perception of SF-36, physical and mental functioning scales as compared with participants without RA [14]. Bivariate analysis shows that five dimensions (PF, RP, RE, MH, and BP) were lower in the RA patients than in the non-RA patients. However, one dimension (GH) was higher in RA patients than in controls. This variability in GH may be due to bias as some controls were taken from those having more severe comorbidities than RA, such as cancer.

In addition, our study shows that RA patients had significantly lower QOL $(p<0.001)$ and lower level of HRQoL in comparison to non-RA conditions. Similarly, RA negatively impacts HRQoL: pooled scores for the physical domains are somewhat lower than the mental health domains, suggesting that RA has a greater impact on physical HRQoL than mental well-being. This finding can be explained by either the evidence of strong family support and a strong social environment among patients or evidence of suppressed feelings among patients. Similar results were found in previous meta-analysis studies $[9,12]$.

Having morning stiffness deteriorates the physical functions as shown in a European study that was conducted on 1061 patients and concluded that morning stiffness reduces the ability to work in patients with RA [20].

Interestingly, the multivariable analysis of QOL and its components shows that the number of times of exercise performed per week has a positive impact on both PCS as well as overall QOL, especially in RA patients. Exercise and sports are important for RA patients for maintaining healthy and strong muscles, preserving joint mobility, and maintaining flexibility. Exercise can also help people sleep well, reduce pain, maintain a positive attitude, and manage weight as stated in an American study [2]. Another study revealed that exercise improves QOL, where participants reported that sport influenced their mental and physical health positively even if they had mobility limitation [17].

The number of cigarettes per day has a positive impact on PCS in the control group in the multivariate analysis. This may be due to the fact that in Lebanon, smoking is a socially acceptable behavior. Smokers may bond together more strongly and create and maintain social relations of their habit. In addition, smokers may present a healthier group of survivors. This selection effect could suggest a positive health for smokers.
Rheumatoid arthritis impact on work can be profound as permanent work disability is common among patients with RA where their ability to meet work demands is limited. Hence, those patients will become jobless as patients who experience at-work productivity loss are prone to develop sick leave, permanent work disability in the future, and low QOL as seen in a cross-sectional study conducted in Amsterdam [11]. Although a Canadian study stated that reduced physical function due to RA is associated with an increased probability of inability to work or absence from work due to RA-associated sickness [6]. In our study, work ability has a positive impact on PCS in both RA and non-RA patients, as well as on MCS and overall QOL in control group suffering from other diseases such as hypertension, cancer, congestive heart failure, myocardial infarction, clinical depression, type 2 diabetes, and so on. This is consistent with a cross-sectional study carried out on 224 employees in the city of Sao Paulo, Brazil, in 2001. All evaluated dimensions of health were significantly associated with work ability $(p<0.0001)$. Results showed that, independently of other characteristics, better physical and mental health are associated with greater work ability [21].

According to the National Arthritis Data Workgroup, pain and stiffness in RA patients last for $>30$ min in the morning or after a long rest [2]. On the other hand, having morning stiffness deteriorates the physical functions as seen in the results of a European study that was conducted on 1061 patients and concluded that morning stiffness reduces the ability to work in patients with RA [20]. Similarly, our study reveals that morning stiffness has a negative impact on both MCS and the overall QOL in RA patients; however, this is not specific to RA patients as it is also valid for non-RA patients where morning stiffness has negative impact on PCS, MCS, and overall QOL.

Finally, the lapse of time between blood transfusion and the onset of RA has a positive impact on MCS of RA patients. This may be due to the fact that RA patients were reassured that the disease was not inoculated to them by blood transfusion.

\section{CONCLUSION}

Rheumatoid arthritis is a common and major health problem in the adult population. This is the first study in Lebanon to assess the QOL of RA patients. RA patients presented lower QOL scores as compared with control. Therefore, it is essential to find medical and social alternatives that have a favorable effect on QOL of RA patients. To improve QOL of patients with RA, this study highlights the importance of campaigns on educating patients, especially with respect to life style habits such as practicing sports regularly and having good work ability, which is important when supporting patients with RA. In addition, finding alternative treatment that reduces morning stiffness will also improve the QOL of RA patients.

This study was a preamble to organize larger studies at the national level in the future seeking to determine risk factors leading to RA and to suggest possible predictors of QOL and finally that will help to further generalize our results.

\section{CONFLICTS OF INTEREST}

The authors declare they have no conflicts of interest. 


\section{AUTHOR CONTRIBUTIONS}

S.A., M.S. and W.B. were responsible for planning and design of the study; M.S. was responsible of data collection; M.S., R.A. were responsible for statistical analysis; S.A., A.A-H., S.R. and S.Z. were involved in the preparation of questionnaire; M.S. wrote the first draft; W.A., S.A. and R.A. provided critical revision. All authors read and approved the final manuscript.

\section{ACKNOWLEDGMENTS}

The authors thank the Lebanese University for supporting this project.

\section{REFERENCES}

[1] Helmick CG, Felson DT, Lawrence RC, Gabriel S, Hirsch R, Kent Kwoh C, et al. Estimates of the prevalence of arthritis and other rheumatic conditions in the United States. Part I. Arthritis Rheum 2008;58;15-25.

[2] van Vugt RM, Rijken PJ, Rietveld AG, van Vugt AC, Dijkmans BA. Antioxidant intervention in rheumatoid arthritis: results of an open pilot study. Clin Rheumatol 2008;27;711-75.

[3] Aurrecoechea E, llorcadiaz J, Diezlizuain ML, Mcgwin G. Jr, Calvo-alen J. Impact of gender in the quality of life of patients with rheumatoid arthritis. J Arthritis 2015;4;160.

[4] Strand V, Khanna D. The impact of rheumatoid arthritis and treatment on patients' lives. Clin Exp Rheumatol 2010;28;S532-S40.

[5] The WHOQOL Group. The World Health Organization Quality of Life assessment (WHOQOL): Development and general psychometric properties. Soc Sci Med 1998;46;1569-85. Available from: https://www.sciencedirect.com/science/article/abs/pii/S 0277953698000094?via\%3Dihub (accessed September, 2017).

[6] Chaaya M, Slim ZN, Habib RR, Arayssi T, Dana R, Hamdan O, et al. High burden of rheumatic diseases in Lebanon: a COPCORD study. Int J Rheum Dis 2012;15;169-76.

[7] Sabbah I, Drouby N, Sabbah S, Retel-Rude N, Mercier M. Quality of life in rural and urban populations in Lebanon using SF-36 health survey. Health Qual Life Outcomes 2003;1;30.

[8] Pollard L, Choy EH, Scott DL. The consequences of rheumatoid arthritis: quality of life measures in the individual patient. Clin Exp Rheumatol 2005;23;S43-S52.
[9] Hays RD, Sherbourne CD, Mazel RM. The RAND 36-item health survey 1.0. Health Econ 1993;2;217-27.

[10] Azevedo AF, Petribú KC, Lima Mde N, Silva AS, Rocha Filho Jde A, Mariano MH, et al. Quality of life of patients with rheumatoid arthritis under biological therapy. Rev Assoc Med Bras 2015;61;126-31.

[11] Bounthavong M, Law AV. Identifying health-related quality of life (HRQL) domains for multiple chronic conditions (diabetes, hypertension and dyslipidemia): patient and provider perspectives. J Eval Clin Pract 2008;14;1002-11.

[12] Haroon N, Aggarwal A, Lawrence A, Aggarwal V, Misra R. Impact of rheumatoid arthritis on quality of life. Modern Rheumatol $2007 ; 17 ; 290-5$.

[13] Martinelli LMB, Mizutani BM, Mutti A, Dèlia MPB, Coltro RS, Matsubara BB. Quality of life and its association with cardiovascular risk factors in a community health care program population. Clinics 2008;63;783-8.

[14] Mili F, Helmick CG, Moriarty DG. Health related quality of life among adults reporting arthritis: analysis of data from the Behavioral Risk Factor Surveillance System, US, 1996-99. J Rheumatol 2003;30;160-6.

[15] Farhat A, Al-Hajje A, Rachidi S, Zein S, Zeid MB, Salameh P, et al. Risk factors and quality oflife of dyslipidemic patients in Lebanon: a cross-sectional study. J Epidemiol Glob Health 2016;6;315-23.

[16] Khalifeh M, Salameh P, Hajje AA, Awada S, Rachidi S, Bawab W. Hypertension in the Lebanese adults: impact on health related quality of life. J Epidemiol Glob Health 2015;5;327-36.

[17] Côté-Leclerc F, Boileau Duchesne G, Bolduc P, Gélinas-Lafrenière A, Santerre C, Desrosiers J, et al. How does playing adapted sports affect quality of life of people with mobility limitations? Results from a mixed-method sequential explanatory study. Health Qual Life Outcomes 2017;15;22.

[18] Matcham F, Scott IC, Rayner L, Hotopf M, Kingsley GH, Norton S, et al. The impact of rheumatoid arthritis on qualityof-life assessed using the SF-36: a systematic review and meta-analysis. Semin Arthritis Rheum 2014;44;123-30.

[19] Corbacho MI, Dapueto JJ. Assessing the functional status and quality of life of patients with rheumatoid arthritis. Rev Bras Reumatol 2010;50;31-43 [Article in English, Portuguese].

[20] Mattila K, Buttgereit F, Tuominen R. Impact of morning stiffness on working behaviour and performance in people with rheumatoid arthritis. Rheumatol Int 2014;34;1751-8.

[21] Martinez MC, Latorre Mdo R. [Health and work ability among office workers]. Rev Saude Publica 2006;40;851-8 [Article in Portuguese]. 